\section{Sound localization and neurons}

Our ability to locate the direction of a sound's source depends largely on differences in the time that it takes for the sound to arrive at each ear. These interaural time delays (ITDs) can be extremely small (about 10 microseconds; ref. 1), so this ability is widely assumed to reflect the combined activity of many neurons ${ }^{2,3}$. Fitzpatrick et al. ${ }^{4}$ estimated the pooling of 40 thalamic neurons to give a resolution of 16 microseconds. Our results, generated by a more conventional method, suggest that human thresholds might be determined by substantially fewer cells.

The ability of a neuron to discriminate between two stimuli is determined by the size of the difference in response relative to the response variability. The receiver operating characteristic ${ }^{5}$ analysis makes it possible to estimate this ability using the same metric as is used to quantify human psychophysical performance. This approach has previously been used to estimate the detection and discrimination of visual neurons ${ }^{6,7}$.

We have applied receiver operating characteristic analysis to previously published results ${ }^{8}$ by assuming that response variability can be described by a Poisson distribution: $\left(\mu^{x} / X !\right) \mathrm{e}^{-\mu}$, where $\mu$ denotes the mean. The percentage of correct responses increases with ITD (Fig. 1b,c).

In psychophysical experiments, it is conventional to define discrimination threshold as the stimulus magnitude at which the psychometric function crosses the $75 \%$ correct level. For the six neurons in the present study, the ITD at which this occurred ranged from $9.9 \mu$ s up to $26.8 \mu$ s (Fig. 1). The lowest of these values is comparable to the thresholds of human observers of about 10 microseconds (ref. 1).

Although these results are based on only six cells, it seems highly likely that there exist neurons with even lower thresholds. If one were to assume that an observer's discrimination threshold reflects the performance of the neuron with the lowest threshold, then one should have to compare the behavioural thresholds to that neuron. The psychophysical thresholds might therefore have been expected to be even lower than 10 microseconds.

These results indicate that single auditory neurons are able to distinguish ITDs as small as the human discrimination thresholds. But they are based on the assumption of a Poisson distribution. There are good reasons for expecting this kind of distribution for response variability ${ }^{9,10}$, especially when only instantaneous variability is con-
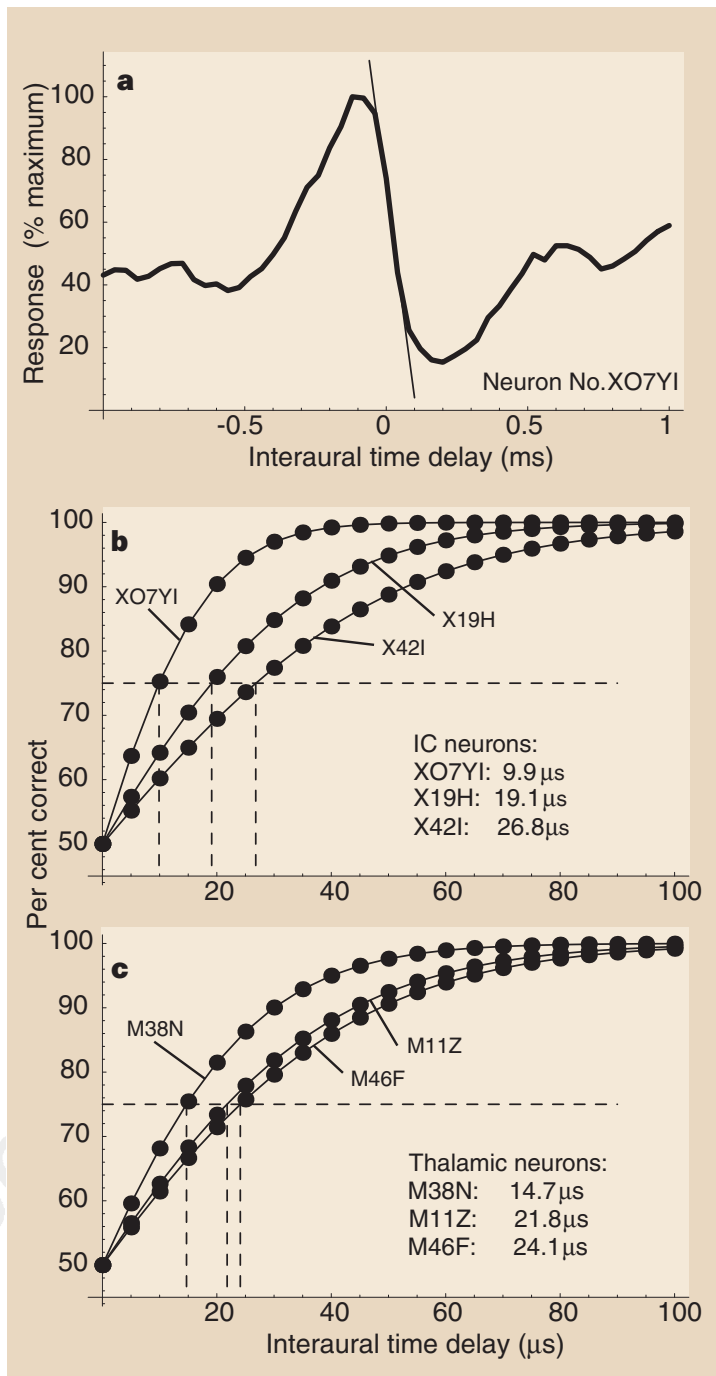

Figure 1 Analysis of interaural time delays (ITDs). a, Response against ITD plot of one inferior colliculus (IC) neuron (redrawn from Fig. 7 of ref. 8, neuron no. X07Y1). The very steep slope at zero ITD makes the neuron translate a small difference in ITD into a large difference in response. b,c, Percentage correct as a function of ITD for the three IC neurons (b) and three thalamic neurons (c). Horizontal broken lines, 75\% correct level; vertical broken lines, ITD at which the functions cross the $75 \%$ correct level. The cell numbers of ref. 8 have been maintained to identify the neurons. The ITD corresponding to each neuron's $75 \%$ correct level is listed. One IC neuron (neuron no. $X O 7 Y 1$ ) has a discrimination threshold comparable to that of human observers.

sidered. It is known that response variability incorporates both an instantaneous and a long-term component ${ }^{6,7}$. In sound localization, it is mainly the instantaneous variability that is relevant.

This might account for part of the large discrepancy between the present results and those of Fitzpatrick et al. . That study combined neurons recorded at different times and from 31 different animals without differentiating long-term from instantaneous variability. This would result in an underestimate of the neurons' capabilities. Also, unlike the present analysis, they did not specifically exploit the steepest slopes of the response against ITD plots (Fig. 1a).

The present results should not be taken to mean that only one neuron is involved in sound localization. The responses of neurons are ambiguous. To remove this ambiguity, the combining of outputs from several neurons might be required. What my results do demonstrate is that it might not be necessary to pool the outputs from many neurons to account for the high accuracy with which human observers can localize sounds.

Bernt Christian Skottun

Skottun Research, 273 Mather Street,
Piedmont, California 94611-5154, USA e-mail:bernt@best.com

1. Mills, A. W. J. Acoust. Soc. Am. 30, 237-246 (1958).

2. Yin, T. C. T. \& Chan, J. C. K. in Auditory Function: Neurobiological Bases of Hearing (eds Edelman, G. M., Gall, W. E. \& Cowan, W. M.) 385-430 (Wiley, New York, 1988).

3. Hall, J. L. J. Acoust. Soc. Am. 37, 814-823 (1965).

4. Fitzpatrick, D. C., Batra, R., Stanford, T. R. \& Kudawa, S. Nature 388, 871-874 (1997).

5. Green, D. M. \& Swets, J. A. Signal Detection Theory and Psychophysics (Krieger, Huntington, NY, 1974).

6. Tolhurst, D. J., Movshon, J. A. \& Dean, A. F. Vision Res. 23, 775-785 (1983).

7. Bradley, A., Skottun, B. C., Ohzawa, I., Sclar, G. \& Freeman, R. D. J. Neurophysiol. 57, 755-772 (1987).

8. Stanford, T. R., Kuwada, S. \& Batra, R. J. Neurosci. 12, 3200-3216 (1992).

9. Rieke, F., Warland, D., de Ruyter van Stevenink, R. \& Bialek, W. Spikes (MIT Press, 1997)

10. Tomko, G. J. \& Crapper, D. R. Brain Res. 79, 405-418 (1974).

scientific correspondence is intended to provide a forum for both brief, topical reports of general scientific interest and technical discussion of recently published material of particular interest to nonspecialist readers. Priority will be given to contributions that have fewer than 500 words, 10 references and only one figure. Detailed guidelines are available on Nature's website or on request from nature@nature.com 